\title{
EMPREGO DE QUÍMICA COMPUTACIONAL NA VERIFICAÇÃO E VALIDAÇÃO DA PRESSÃO DE DETONAÇÃO DE EXPLOSIVO PLÁSTICO-PBX
}

\author{
Fausto B. Mendonça a, Rene F. B. Gonçalves ${ }^{a}$, Girum S. Urgessa ${ }^{b}$, Koshun Iha ${ }^{a}$, Marcela Domingues ${ }^{a}$ e José A. F. F. Rocco ${ }^{a}$ * \\ aDepartamento de Química, Instituto Tecnológico de Aeronáutica, 12228-900 São José dos Campos - SP, Brasil \\ ${ }^{\mathrm{b}} \mathrm{Sid}$ and Reva Dewberry Department of Civil, Environmental and Infrastructure Engineering, George Mason University, 22030, \\ Fairfax - VA, United States of America
}

Recebido em 03/10/2017; aceito em 14/11/2017; publicado na web em 11/12/2017

\begin{abstract}
COMPUTATIONAL CHEMISTRY EMPLOYMENT IN VERIFICATION AND VALIDATION OF DETONATION PRESSURE OF PLASTIC EXPLOSIVE - PBX. Prediction of chemical explosions parameters is an important step for blast tests for civil and military applications. Applying computational chemistry and experimental results, this paper presents the decay rate of pressure in air from the epicenter of $2.70 \mathrm{~kg}$ of PBX-Plastic bonded explosive detonation to the distance of 2.0 meters. Pressure in the epicenter was calculated using reactive molecular dynamics simulations and the incident pressure at $1.3 ; 1.6$ and 2.0 meters were measured with piezoelectric pressure sensors. The explosive for the full-scale test were non-confined in a cylindrical shape. Results of simulation and recorded values were consolidated and the rate of pressure decay was verified by statistic regression. Good agreement was verified between computational and experimental pressures data. This research can help designers to prepare protection devices in test areas, storages of explosives or important buildings for military proposes.
\end{abstract}

Keywords: molecular dynamic simulation; chemical explosion; incident pressure; plastic bonded explosive.

\section{INTRODUÇÃO}

Explosão química pode ser definida como uma reação exotérmica que libera instantaneamente alta quantidade de energia, geralmente por uma grande quantidade de calor e gás, para o meio sob velocidade altíssima, em geral supersônica. ${ }^{1,2}$ A dissipação dessa energia para o meio pode ser sob a forma de onda de choque, no ar, trincas, em rochas ou sólidos e bolhas em água; propulsão de estilhaços e emissão de energia térmica também estão associadas à dissipação da energia de uma explosão química. ${ }^{3}$ A reação exotérmica que ocorre sob altíssima velocidade na explosão química gera gases e vapor; como o volume em que o material encontra-se não pode se expandir no curto intervalo de tempo que a reação ocorre, a pressão eleva-se a valores de centenas de atmosferas, gerando, assim, uma onda de choque de pressão que causará danos às paredes do invólucro e aos objetos e pessoas próximos. ${ }^{4}$ Este trabalho está focado na dissipação dessa energia no ar atmosférico. Os parâmetros associados a uma explosão, como sobre pressão, impulso e tempo de duração são elementos importantes para se avaliar os danos causados por explosivos. A onda de choque causada pela detonação de um alto explosivo move-se em todas as direções e se expande sob um formato esférico. Porém, ao atingir certa distância do epicentro torna-se plana. ${ }^{5} \mathrm{O}$ artigo apresenta a simulação da pressão gerada no epicentro da detonação de $3,0 \mathrm{~kg}$ de PBX, com o auxílio do pacote computacional LAMMPS, e os resultados da pressão medida a 1,3; 1,6 e 2,0 m de distância do explosivo em ensaio experimental de campo com o mesmo explosivo. Foi verificada a taxa de decaimento da pressão partindo do epicentro até uma distância de 2,0 m. O uso de simulação computacional aplicado à dinâmica de moléculas é frequentemente encontrado na literatura bem como o uso do pacote computacional LAMMPS. ${ }^{6-8}$

\section{Explosivo químico}

Muito tem-se pesquisado na área de explosivos químicos. ${ }^{9-12}$ As

\footnotetext{
*e-mail: friz@ita.br
}

substâncias classificadas como explosivos possuem, em geral, na sua composição, nitrogênio, oxigênio e/ou outros oxidantes. ${ }^{2} \mathrm{O}$ oxigênio e o nitrogênio costumam estar associados. Algumas substâncias fogem a essa regra como é o caso da azida de chumbo e alguns compostos nitrogenados que não contêm oxigênio. ${ }^{3}$ Na reação química que gera a explosão, o oxigênio e o nitrogênio se separam unindo-se ao componente que faz o papel de combustível, como no caso do carbono ou do hidrogênio. Essa reação libera altas quantidades de energia e calor em forma de gases. O calor liberado nessa reação é a diferença entre o calor requerido para quebrar a ligação original e o calor liberado na união do novo composto. ${ }^{13}$

Os explosivos químicos podem ser classificados quanto sua natureza química em explosivos ou misturas explosivas e quanto sua performance durante a queima em primário e secundário. ${ }^{14}$

As substâncias que se classificam como explosivos, ou seja, que liberam grandes quantidades de gases e calor em curto espaço de tempo, apresentam grupos terminais com propriedades tais como: compostos nitrados; esteres nitrados; azidas; nitraminas; derivados de ácidos clóricos ou perclóricos, além de outros compostos capazes de gerar explosões, como, tetrazinas e peróxidos. A classificação quanto à performance indica a sensibilidade do explosivo a impacto ou calor para iniciar sua detonação. O explosivo classificado como primário é menos estável que o secundário, sendo ignitado com menor energia (calor ou impacto), porém, em geral, possui menor capacidade de gerar dano. O explosivo secundário é mais estável, não podendo ser detonado com calor e impacto, entretanto possui maior capacidade de gerar dano, sendo denominado alto explosivo., ${ }^{2,15}$ Em função destas características, o explosivo primário é utilizado para iniciar a detonação do explosivo secundário, formando assim o trem explosivo. ${ }^{9,16}$ A onda de choque formada pela detonação do explosivo primário é que gera a detonação do explosivo secundário. Os propelentes são materiais combustíveis que possuem todo o oxidante necessário para sua queima em sua composição. Queimam de forma controlada, evitando o processo de explosão, em geral apresentam chama ou centelhas e sons de fissurações intensas, mas não caracterizam uma detonação (explosão). 
São ignitados por centelha ou chama e transformam-se de sólido para gás em frações de milissegundos. ${ }^{17}$

O HMX, denominado Ciclotetrametileno Tetranitroamina $\left(\mathrm{C}_{4} \mathrm{H}_{8} \mathrm{~N}_{8} \mathrm{O}_{8}\right)$, cuja sigla em inglês significa High Melting point Explosive, possui massa molar de 296,2 $\mathrm{g} \mathrm{mol}^{-1} \mathrm{e}$ velocidade de detonação de $9.000 \mathrm{~m} \mathrm{~s}^{-1}$. Encontra-se em quatro fases polimórficas, $\alpha, \beta$, $\gamma$ e $\delta$, dependendo das condições de cristalização em seu processo de produção. Estas formas vão apresentar diferenças entre si em relação às densidades e sensibilidade a impactos. O HMX é não higroscópico e insolúvel em água, sendo a fase $\beta$ a menos sensível a impacto e, por isso, é utilizada como explosivo secundário em artefatos bélicos.

\section{Pressão de uma explosão}

Uma onda supersônica é gerada na detonação de um alto explosivo junto ao ponto de iniciação e se desloca no meio através do explosivo, sustentada pela decomposição exotérmica do material que vem logo atrás. Essa onda de choque, além de se propagar no meio onde foi gerada, também retroalimenta o material explosivo, aplicando uma pressão que contribui na cinética química da decomposição do explosivo. A onda de choque provocará no meio onde ocorre a detonação resultados tais como: trincas em rochas, crateras em solos, bolhas em ambientes aquáticos. ${ }^{18}$ A energia associada ao evento dependerá do volume de gases e do calor gerados pela explosão. A pressão de explosão é a pressão máxima atingida por uma massa de explosivo queimada num volume determinado. Uma correção na lei do gás ideal se faz necessária para a determinação dessa pressão por meio do co-volume $\alpha$ conforme Equação 1, sendo que este co-volume corresponde ao volume de vazios entre as moléculas. $\mathrm{V}_{\mathrm{i}}$ corresponde ao volume inicial, $\mathrm{n}$ ao número de moles e $\mathrm{T}_{\mathrm{e}}$ a temperatura da explosão em Kelvin, R é a constante dos gases.

$$
P_{e}\left(V_{i}-\alpha\right)=n R T_{e}
$$

A força exercida pelos gases na explosão de um alto explosivo pode ser expressa em $\mathrm{kJ} \mathrm{g}^{-1}$ ou $\mathrm{MJ} \mathrm{kg}^{-1}$ e a Equação 2 fornece esse valor. $^{18}$

$$
F=n R T_{e}
$$

A equação de vapor de pressão Clausius-Clapeyron vem de relações termodinâmicas macroscópicas que usam a energia livre de Gibbs sob duas condições: primeiro considerando a densidade do vapor muito menor que a do líquido e, segundo, a densidade do vapor é suficientemente baixa para poder ser aplicada à equação de estado do gás ideal. Essas considerações são válidas para substâncias comuns próximo ao ponto de fusão e a equação Clausius-Clapeyron fornece a relação de temperatura e pressão da Equação 3, em que A' representa o fator pré-exponencial da equação de vapor de pressão Clausius-Clapeyron para líquido, L é o calor latente da vaporização do líquido na temperatura de fusão $\left(\mathrm{T}_{\mathrm{f}}\right), \mathrm{T}$ a temperatura e $\mathrm{R}$ a constante universal dos gases.

$$
p=A^{\prime} e^{\frac{-L}{R T}} \text { ou } \ln p=\frac{a_{1}}{T}+a_{0}
$$

Entretanto, quando a temperatura de fusão aumenta até a temperatura crítica, a Equação 3 não se aplica, pois fornecerá valores de pressão inconsistentes. A equação de Antoine, que é resultado do aperfeiçoamento da equação de Augusto, é apresentada como Equação 4, sendo A o fator de frequência na equação de Arrhenius.

$$
\log _{10} p=A-\frac{B}{T}
$$

Para temperatura $\mathrm{T}$ em Kelvin o fator $\mathrm{C}$ assume valores negativos; por isso a equação sugere que a temperatura de zero absoluto seja um valor acima do conhecido. Dessa forma, faz com que se questione a eficácia da equação. Sua aplicação pode ser feita apenas para temperaturas entre a de fusão e de queima. Equações sobre vapor de pressão de água foram exaustivamente estudadas e desenvolvidas, como a equação de Goff-Gratch, porém não podem ser aplicadas para qualquer substância devido sua limitação de faixa de temperatura. Dessa forma, faz-se necessário o desenvolvimento de uma equação para tratar da pressão gerada pelos gases de materiais energéticos. Usando valores constante para $\mathrm{a}_{0}$ e A' na Equação 3, considerando $\mathrm{p}=1$ bar e considerando a falta de valores de pressão que não estejam próximos da temperatura de fusão, chega-se à Equação 5, em que $\mathrm{p}_{\mathrm{f}}$ é a pressão de vapor na temperatura de fusão.

$$
a_{0}=\ln A^{\prime}=\ln p_{f}+\frac{L}{R T_{f}}
$$

Extrapolando $p_{\mathrm{f}}$ pela equação de pressão de vapor de sublimação do sólido obtém-se a Equação 6.

$$
p=A_{s}^{\prime} e^{-L_{S \rightarrow G} / R T}=>p_{f}=A_{s}^{\prime} e^{-L_{S \rightarrow G} / R T_{f}}
$$

Essas extrapolações podem culminar em erros na estimação da temperatura de queima. Entretanto, essa solução traz um valor razoável, uma vez que o calor latente para a mudança de fase de sólido para gasoso não é tão sensível à temperatura quanto a mudança de líquido para gasoso, podendo, assim, ser considerado constante.

\section{PARTE EXPERIMENTAL}

\section{Ensaio experimental}

Para os testes experimentais foram utilizados dez corpos de prova de massa 2,7 kg de PBX (Plastic Bonded Explosive) 80/20, não confinados, com dimensões de $20 \mathrm{~cm}$ de altura por $10,5 \mathrm{~cm}$ de diâmetro, em formato cilíndrico. Todas as detonações ocorreram na área de testes do Departamento de Ciência e Tecnologia Aeroespacial (DCTA). O fator de forma do explosivo foi fixado como uma seção cilíndrica com o objetivo de simular a cabeça de guerra de um armamento. ${ }^{19}$ A detonação foi iniciada na parte superior do explosivo. A Figura 1 apresenta os corpos de prova dos explosivos estocados, nos quais é possível notar os reforçadores já posicionados na parte superior do corpo de prova.

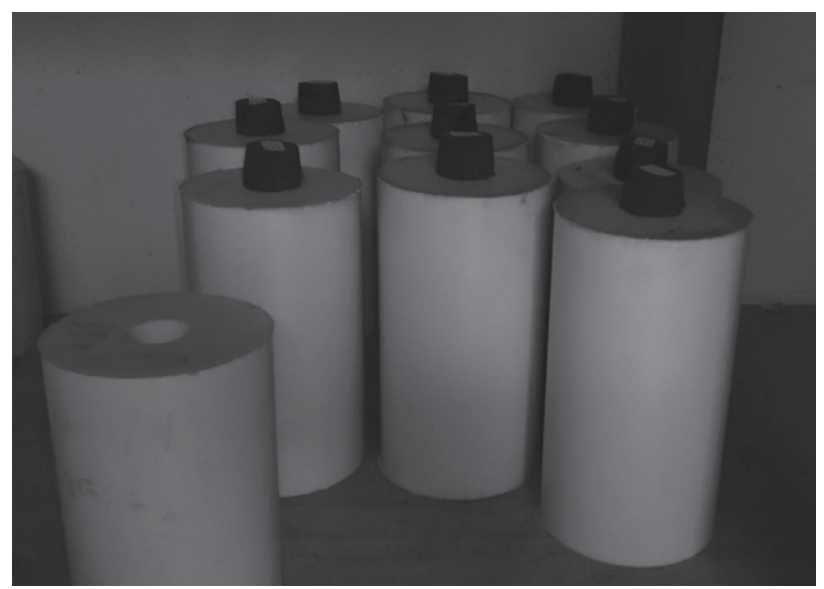

Figura 1. Corpos de prova do PBX em formato cilíndrico

O trem explosivo foi montado numa cavidade na parte superior do cilindro, conforme visualizado na Figura 2. O explosivo foi disparado por uma espoleta elétrica comandada a uma distância de $250 \mathrm{~m}$ de um 
abrigo onde a equipe se protegeu durante os ensaios experimentais. Dois sensores de pressão piezoelétricos foram posicionados a 1,3 metros e três a 1,6 e 2,0 metros da posição do explosivo.

Os sensores de pressão possuíam capacidade de leitura até 5,0 $\mathrm{MPa}$ e tempo de reamostragem de leitura de $10 \mu$ s. O uso de explosivo não confinado teve por objetivo evitar a geração de fragmentos, impedindo, assim, influências secundárias nas medição de pressão exercidas pela passagem da onda de choque.

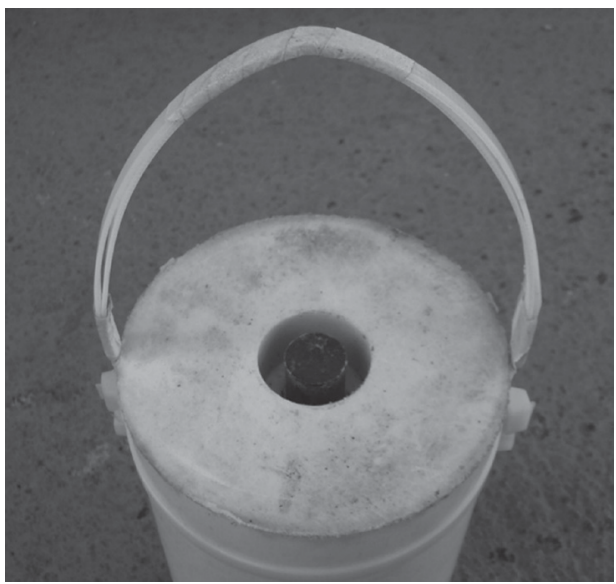

Figura 2. Cavidade na parte superior do corpo de prova cilindrico, destinadoao à instalação do explosivo primário

Os sensores foram posicionados nas distâncias já mencionadas e apontavam diretamente para o explosivo. A Figura 3 apresenta um desses sensores imediatamente antes da detonação do PBX.

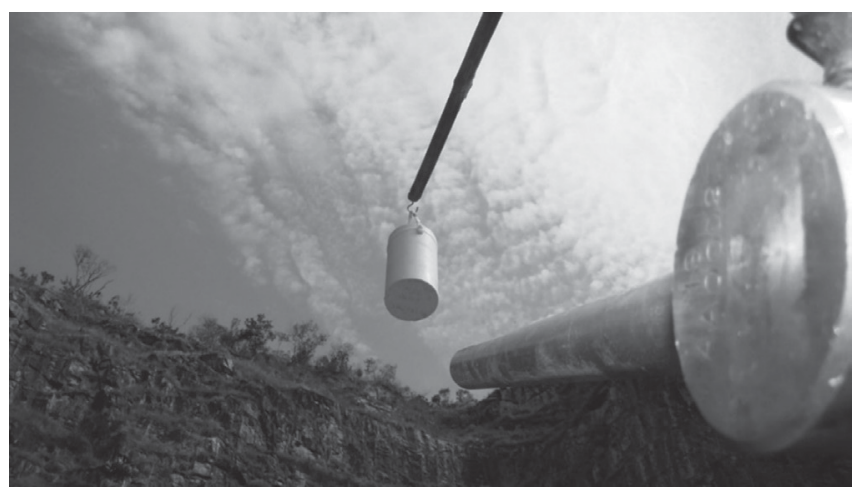

Figura 3. Sensor piezelétrico apontando para o explosivo

Os valores de pressão anotados foram relacionados ao valor simulado no epicentro para possibilitar a proposta de uma função matemática que indicasse a taxa de decaimento da pressão, partindo do epicentro até as distâncias onde foram anotadas para cada experimento (R). A Tabela 1 apresenta os valores de massa em equivalente TNT - Trinitrotolueno (W), distância real (R), distância em escala (Z). O equivalente em TNT é um método de quantificação da energia libertada em explosões. A tonelada (ou ton) de TNT é uma unidade de energia igual a 4,184 gigajoules, aproximadamente a quantidade de energia liberada pela detonação de uma tonaleda de TNT. A megatonelada (ou megaton) de TNT é uma unidade de energia equivalente a 4,184 petajoules.

\section{Simulação computacional}

As simulações de dinâmica molecular reativa (RMD) foram realizadas com a fase $\beta$ do HMX, com densidade experimental de 1,91
Tabela 1. Valores de R, W, Z

\begin{tabular}{cccc}
\hline Teste & $\mathrm{R}(\mathrm{m})$ & $\mathrm{W}(\mathrm{kg})$ & $\mathrm{Z}\left(\mathrm{m} / \mathrm{kg}^{1 / 3}\right)$ \\
\hline 1 & 1,30 & 2,759 & 0,927 \\
2 & 2,00 & 2,718 & 1,433 \\
3 & 2,00 & 2,714 & 1,434 \\
4 & 2,00 & 2,692 & 1,437 \\
5 & 2,00 & 2,585 & 1,457 \\
6 & 2,00 & 2,723 & 1,432 \\
7 & 2,00 & 2,606 & 1,453 \\
8 & 2,00 & 2,761 & 1,425 \\
9 & 2,00 & 2,721 & 1,432 \\
10 & 1,60 & 2,603 & 1,163 \\
\hline
\end{tabular}

$\mathrm{g} \mathrm{cm}^{-3}$. Os parâmetros de célula unitária foram obtidos na literatura

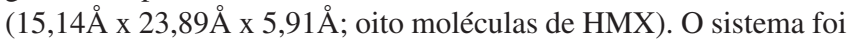
inicialmente minimizado utilizando baixa temperatura (5K), a fim de promover o relaxamento das posições atômicas (Fig. 4). Utilizando o modelo ReaxFF, o sistema evoluiu de acordo com um ensemble NVE, controlada por um termostato de Berendsen. Para detonação e evolução do sistema, a temperatura foi ajustada de 300 a $2000 \mathrm{~K}$ com constante de dumping de temperatura de $100 \mathrm{fs}$. As simulações descritas foram realizadas empregando-se o pacote computacional LAMMPS.
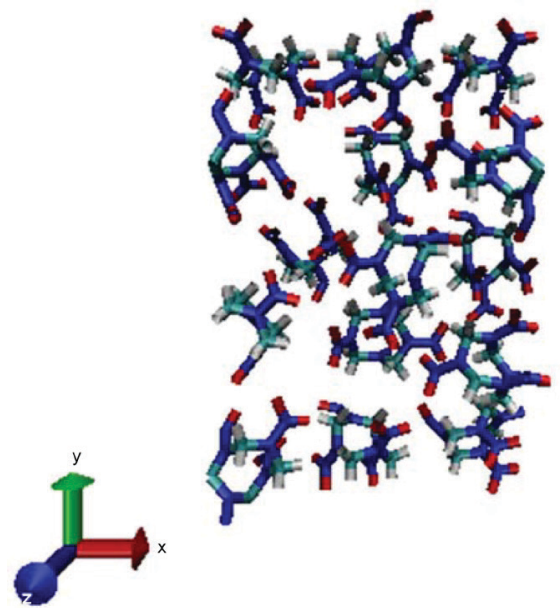

Figura 4. Célula unitária do $\beta-H M X$ (legenda de cores: $a z u l=N$; vermelho $=O ;$ ciano $=C ;$ branco $=H$ )

Como o processo envolve uma detonação (combustão de alta velocidade), o passo da simulação foi mantido em $0,1 \mathrm{fs}$, a fim de prever rápidos deslocamentos das espécies no domínio considerado.

\section{RESULTADOS E DISCUSSÃO}

Na simulação de dinâmica molecular reativa, as ordens de ligação são continuamente analisadas conforme as interações progridem, possibilitando quebra e formação de ligações contínuas, de acordo com as variáveis físicas do sistema. Como observado na Figura 5, o sistema apresenta, inicialmente, variação nas distâncias relativas entre as moléculas de HMX, indicando a possibilidade de ocorrência de processo de fusão do material, com pequena quantidade de outras espécies se desprendendo.

Conforme avanço do tempo, as ligações internas das moléculas de HMX começam a se quebrar, formando diversos tipos de fragmentos 
e espécies intermediárias. A partir disso, os átomos começam a se reorganizar em novas espécies químicas, menores e mais estáveis, aproximando-se cada vez mais dos produtos principais da decomposição térmica do material (água e dióxido de carbono). Assim, observa-se que após 40 ps, há aumento da quantidade de espécies gasosas formadas ( $\mathrm{CO}, \mathrm{CO}_{2}, \mathrm{CH}_{2}, \mathrm{CH}, \mathrm{CH}_{2} \mathrm{O}$ etc.), assim como a dispersão destas espécies pelo volume de controle considerado, indicando também um aumento expressivo da pressão interna do sistema, conforme é possível observar na Figura 6. O tempo total da simulação dinâmica molecular reativa foi de 57 h $04 \mathrm{~m} 44 \mathrm{~s}$. A quantificação das espécies geradas consta na Figura 7 e a entalpia envolvida no processo foi calculada pela diferença entre estado final e inicial da seguinte forma: $\Delta \mathrm{H}=-35702,299-(-33229,877)=$ $-2472,422 \mathrm{kcal} / \mathrm{mol}$.

A presença de valores negativos de pressão indica constrição instantânea do sistema, ou seja, ao longo do tempo, as diversas moléculas do sistema e vizinhanças (sistema periódico) se difundem pelo meio, trocando espécies e energia. A Figura 6 apresenta a evolução da pressão do sistema no volume de controle considerado o cristal de HMX (célula unitária).

Durante este processo (em 50 ps) a pressão média do sistema atingiu um valor médio de 1248 atmosferas (124,8 MPa). Este valor é condizente com a realidade experimental, uma vez que foi considerada uma célula unitária contendo 375 átomos, submetida a um processo de rápida decomposição térmica (detonação).

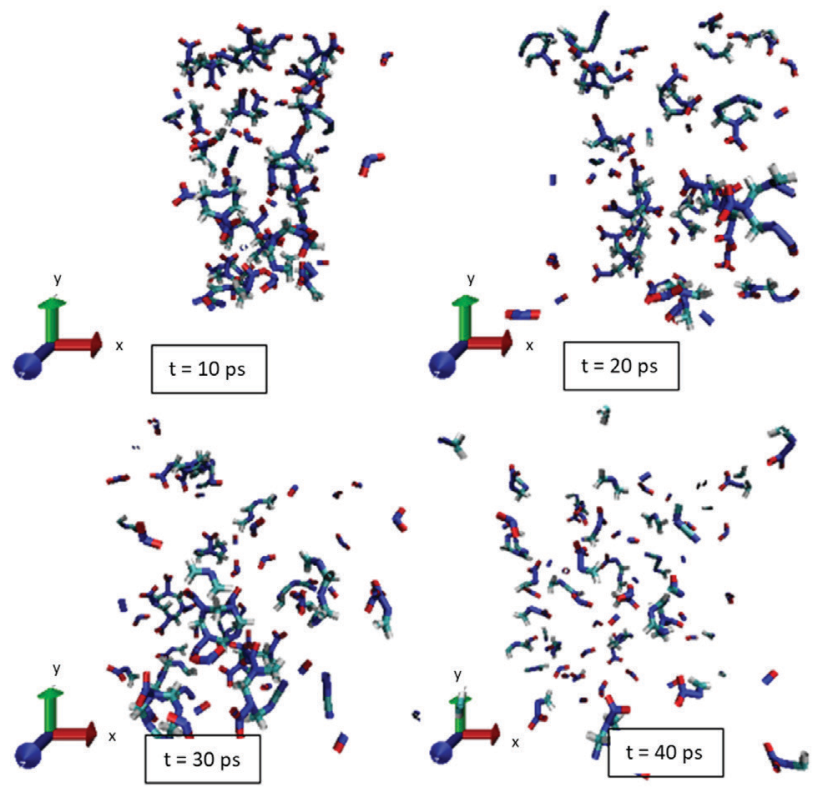

Figura 5. Evolução da decomposição térmica do cristal de HMX conforme avanço do tempo após estímulo da detonação

Com relação à energia do sistema, durante o tempo da simulação a variação observada foi de, aproximadamente, $818 \mathrm{kcal} \mathrm{mol}^{-1}$, ou $2.76 \mathrm{kcal} \mathrm{g}^{-1}$. Considerando o valor experimental de entalpia dos produtos de detonação do HMX (1.48 $\left.\mathrm{kcal} \mathrm{g}^{-1}\right)$, infere-se que a modelagem molecular realizada apresenta proximidade significativa e, consequentemente, boa precisão quanto aos resultados obtidos. As medições de pressão dos sensores, posicionados conforme descrito no item "ensaio experimental", são apresentadas na Tabela 2.

Para que uma proposta de função fosse gerada, foi necessário obter a média dos valores das leituras tomadas sob as mesmas distâncias, esses valores constam na Tabela 3.

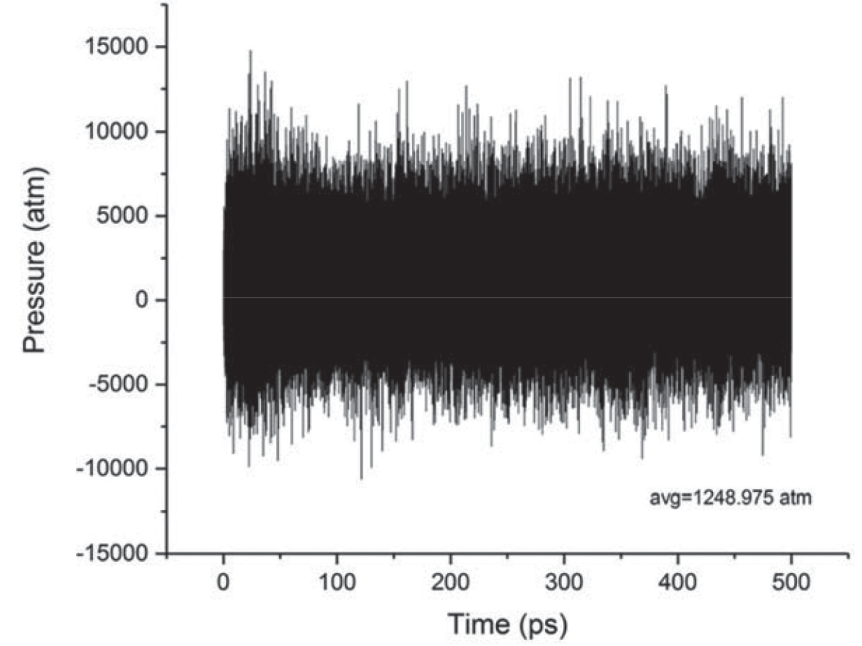

Figura 6. Evolução da pressão do sistema baseado na difusão/dispersão das espécies químicas no volume considerado

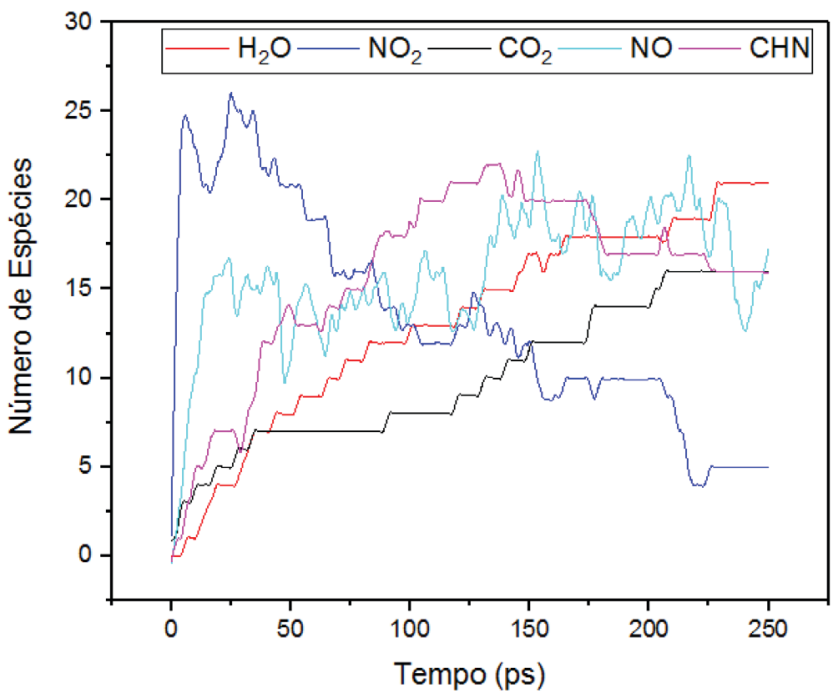

Figura 7. Quantificação da formação das espécies durante a simulação

Tabela 2. Medições de pressão

\begin{tabular}{ccccc}
\hline \multirow{2}{*}{ Teste } & \multirow{2}{*}{$\begin{array}{c}\text { Distância de } \\
\text { leitura }(\mathrm{m})\end{array}$} & \multicolumn{3}{c}{ Pressão medida (MPa) } \\
\cline { 3 - 5 } & 1,3 & 0,844 & 1,997 & Sensor 1 \\
\hline 2 & 2 & 0,718 & 1,303 & - \\
3 & 2 & 0,496 & 0,881 & - \\
4 & 2 & 0,755 & 0,986 & - \\
5 & 2 & 0,937 & 1,497 & 0,933 \\
6 & 2 & 0,307 & 1,071 & 0,413 \\
7 & 2 & 0,495 & 1,543 & 0,956 \\
8 & 2 & 0,596 & 1,296 & 0,763 \\
9 & 2 & 0,442 & 1,216 & 0,746 \\
10 & 1,6 & 0,538 & 0,971 & 2,472 \\
\hline
\end{tabular}

Esses dados permitiram a construção de uma função que indicasse a taxa de decaimento da pressão entre as distancias de 1,3 a 2,0 metros, por meio de inserção de linha de tendência exponencial, Região C da Figura 8. O coeficiente de determinação $\left(\mathrm{R}^{2}\right)$ indica que o modelo explica $90,25 \%$ da variabilidade dos dados. ${ }^{20}$ Entretanto, a função 
Tabela 3. Valores médios de pressão para cada distância da posição do explosivo

\begin{tabular}{cc}
\hline Distância $(\mathrm{m})$ & Média da pressão medida/simulada $(\mathrm{MPa})$ \\
\hline 0 & 124,80 \\
1,3 & 1,42 \\
1,6 & 1,33 \\
2,0 & 0,87 \\
\hline
\end{tabular}

não pode ser estendida para a Região B, pois a queda acentuada da pressão registrada no epicentro até a distância de 1,3 metros indica que ocorre um comportamento diferente. Esse comportamento pode ser explicado pela resistência oferecida pelo ar ao deslocamento da onda de choque. Na Região A consta a informação gerada pela simulação, anteriormente apresentada na Figura 6. Nota-se que a escala do eixo horizontal nessa região está deformada para permitir a visualiação da variação da pressão no eixo vertical. $O$ valor 0 (zero) no eixo horizontal indica o epicentro da detonação.

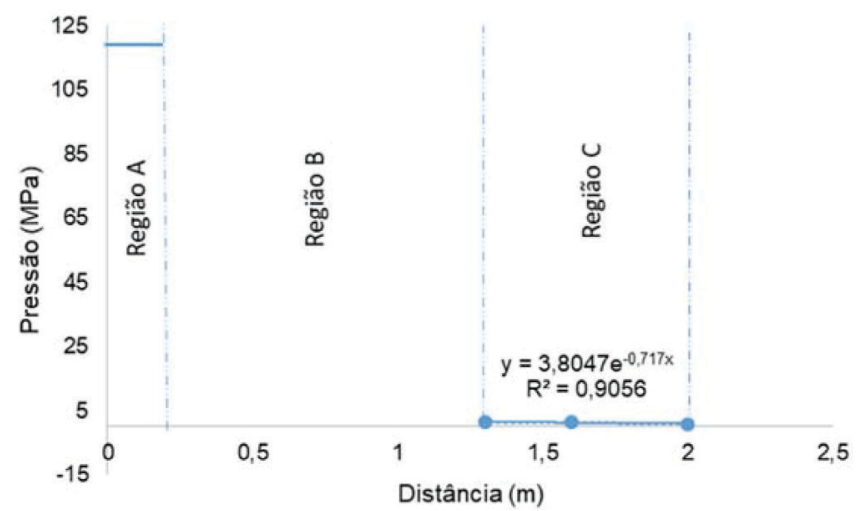

Figura 8. Função obtida por regressão linear para explicar a taxa de decaimento da pressão

A Região B necessita de informação sobre o decaimento da pressão que é fornecido pela resistência do ar atmosférico ao deslocamento da frente de onda de choque, podendo-se afirmar que o decaimento é da ordem de $98 \%$.

\section{CONCLUSÃO}

A energia desenvolvida na explosão de um material energético é determinada pelas propriedades dos componentes desse material e suas composições. Além disso, essas propriedades podem sofrer alterações durante liberação de calor na decomposição térmica e na explosão. No intuito de auxiliar na determinação dessas propriedades foi verificado o comportamento do HMX- $\beta$ durante sua detonação por meio de simulações de dinâmica molecular reativa. O sistema evoluiu de acordo com um ensemble NVE, controlado por um termostato de Berendsen. Para detonação e evolução do sistema, a temperatura foi ajustada de 300 a $2000 \mathrm{~K}$ com constante de dumping de temperatura de 100 fs. As simulações descritas foram realizadas empregando-se o pacote computacional LAMMPS. O comportamento do material na deflagração é de suma importância no estudo da violência da detonação. Medições de pressão verificadas em testes em escala com explosivo PBX 80/20 permitiram relacionar as pressões desenvolvidas no epicentro, dadas pela simulação, com pressões registradas próximo ao explosivo não confinado. Esses dados permitiram propor uma equação de comportamento da pressão desenvolvida durante a detonação entre 1,3 e 2,0 metros. Foi possivel verificar o valor da pressão desenvolvida no epicentro através de simulação computacional. O decaimento da pressão desde o epicentro até o primeiro ponto de leitura a $1,3 \mathrm{~m}$ do explosivo foi de $98 \%$.

\section{REFERÊNCIAS}

1. Sabatini, J. J.; Wingard, L. A.; Guzman, P. E.; Johnson, E. C.; Drake, G. W.; Bis-Isoxazole dinitrate: A potential propellant and explosive ingredient, $42^{\text {nd }}$ International Pyrotechnics Society Seminar, Grand Junction, 2016.

2. Oxley, J. C. In Explosive Effects and Applications, $1^{\text {st }}$ ed., Zukas, J. A., Walters, W. P., eds.; Springer Science: New York, 2003, pp. 137-172.

3. Lee, P. R.; In Explosive Effects and Applications, $1^{\text {st }}$ ed., Zukas, J. A., Walters, W. P., eds.; Springer Science: New York, 2003, pp. 23-46.

4. Silva, W. C. L.; Tese de Mestrado, Instituto Tecnológico de Aeronáutica, Brasil, 2007.

5. Dharma Rao, V.; Srinivas Kumar, A.; Venkateswara Rao, K.; Krishna Prasad, V. S. R.; Propellants, Explos., Pyrotech. 2015, 40, 138.

6. Kim, S.; Lee, J.; Jo, S.; Brooks, C. L.; Lee, H. S.; Im, W.; J. Comput. Chem. 2017, 21, 1879.

7. Lesch, V.; Diddens, D.; Bernardes, C. E. S.; Golub, B.; Dequidt, A.; Zeindlhofer, V.; Sega, M.; Schröder, C.; J. Comput. Chem. 2017, 9, 629.

8. Ridgway, H. F.; Orbell, J.; Gray, S.; J. Membr. Sci. 2017, 524, 436.

9. da Silva, G.; Nakamura, N. M.; Iha, K.; Quim. Nova 2008, 31, 2060.

10. Kirchhof, E.; Rocha, R. J.; Nakamura, N. M.; Lapa, C. M.; Pinheiro, G. F. M.; Gonçalves, R. F. B.; Rocco, J. A. F. F.; Iha, K.; Quim. Nova 2016, 39,661 .

11. Rocha, R. J.; Lima, J. E. S.; Gomes, S. R.; Iha, K.; Rocco, J. A. F. F.; Quim. Nova 2013, 36, 793.

12. Silva, G.; Mattos, C.; Nakamura, M.; Iha, K.; Quim. Nova 2004, 27, 889.

13. Cooper, P. W.; Explosive Effects and Applications, $1^{\text {st }}$ ed., Zukas, J. A., Walters, W. P., eds.; Springer Science: New York, 2003, pp. 115-135.

14. Kim, B.; Jang, S.; Yoh, J. J.; Comput. Fluids 2017, 156, 368.

15. Oyler, K. D.; Metha, N.; Savithra, G. H. L.; Winter, C. H.; Lead-free primary explosives and beyond, $42^{\text {nd }}$ International Pyrotechnics Society Seminar, Grand Junction, 2016.

16. Kim, B.; Kim, M.; Yoh, J. J.; Proc. Combust. Inst. 2017, 36, 2699.

17. Manea, S.; Manea, G. K. B.; Iha, K.; Rocco, J. A. F. F.; Quim. Nova 2014, 37, 27.

18. Akhavan, J.; The Chemistry of Explosives, $2^{\text {nd }}$ ed., RSC: Cambridge, 2004.

19. Anastacio, A. C.; Knock, C.; Propellants, Explos., Pyrotech. 2016, 41, 682.

20. Montgomery, D. C.; Runger, G. C.; Estatística Aplicada e Probabilidade para Engenheiros, $4^{\text {th }}$ ed., LTC: Rio de Janeiro, 2009. 